\title{
Statics Simulation of the Buffer Wastewater Neutralization Process
}

\author{
Ruslan A. Osipa ${ }^{\mathrm{a}}$ \\ ${ }^{a}$ Department of Chemical Production Automation, National Technical University of Ukraine "Igor Sikorsky \\ Kyiv Polytechnic Institute”, Kyiv, Ukraine
}

Received: 01 March 2019; Accepted: 07 June 2019; Published: 08 July 2019

\begin{abstract}
The $\mathrm{pH}$ value is a very common chemical measurement and is an indicator of water quality. For dilute solutions of strong acids and bases, the $\mathrm{pH}$ is related to their concentration, and therefore, it's related to the required for neutralization dose of the reagent, a clear dependence. In this case, the $\mathrm{pH}$ value is quite reliable parameter to control the process of neutralization. However, when the wastewater components that have buffer properties, for example, mixtures of weak acids and bases and their salts in mixture with strong acids are present, the neutralization process is described already by a group of characteristics. In the last case, traditional automatic systems cannot cope with the qualitative $\mathrm{pH}$ regulation.

The article is devoted to the mathematical statics model of a neutralization buffer of wastewater process development. Based on the formulated assumptions, this process was divided into stages. After considering the characteristics of each stage the mathematical model of the process was obtained. The theoretical research results are specified in the developed calculation algorithm of the total reagent dose which is required to neutralize the wastewater, which have strong and weak acids in its composition. The theoretical and practical research results were approbated in the design of automatic control system of the buffer wastewater neutralization process in the enterprise of low-waste technology.
\end{abstract}

Index Terms: Buffer wastewater, $\mathrm{pH}$, static characteristic of the neutralization process, mathematical model of static of the neutralization process, reagent, titration of a strong acid, titration of a weak acid, automated control system (ACS) of buffer wastewater neutralization process.

(C) 2019 Published by MECS Publisher. Selection and/or peer review under responsibility of the Research Association of Mode rn Education and Computer Science

\footnotetext{
* Corresponding author.

E-mail address: ruslanosipa@gmail.com
} 


\section{Introduction}

Nowadays the task of water conservation has become extremely important in the countries of the European Union as well as in the whole world [1]. The pollution by dumping of harmful substances into the environment, groundwater and soil in the area of industrial companies and airports refers to the factors of the adverse human activity effects on the environment $[2,3]$.

The treatment of wastewater is almost always a complex of methods. A combination of mechanical treatment, neutralization of waste, chemical and bioremediation is very widely used. The analysis of the leading specialists in this sector $[4,5,6]$ have shown the neutralization process is very common for the wastewater treatment. This is due to the fact that acids and alkalis have a considerable specific weight among the pollutants. The typical purification processes in general and the neutralization process in particular are characterized by nonstationarity. The fact is that the quantitative and qualitative wastewater composition varies during the day, week, month. In addition, volley discharges of wastewater often occur as well. Therefore, the process of neutralization will be effective in terms of the automated control system functioning, the mail task of which will be to find and implement optimal control actions after the input parameters disturbance.

The $\mathrm{pH}$ value can be used as controlled variable, as a regulated variable, as an indicator of treated water quality $[7,8,9,10]$. There are many aspects that contribute to the problem of $\mathrm{pH}$ measurement, including understanding of what $\mathrm{pH}$ actually is. This is very important in the mathematical modeling of the processes under consideration. In order to understand the principles of modeling, measurement and $\mathrm{pH}$ control better, we need to know the basic properties of liquids, ionic reactions and what is their relationship to the $\mathrm{pH}$ value. That is why the process of neutralization buffer wastewater was divided into stages in the beginning, which are described on the basis of reaction chemistry.

The task of $\mathrm{pH}$ controlling is a good demonstration of the weakest link law (in this case, in the management of one of the sewage treatment processes). The fact is we forget the nature of the controlled process very often. For nonlinear neutralization process sensor measurements work well only in some operating ranges, and within a short period of time. Obviously, based on bad process parameters readings, quality regulation is impossible. For a very nonlinear process of regulation, the executive mechanism must accurately realize both very small and large fluctuations in the contamination ingredient. And very many drives do not cope with this task $[11,12]$.

Static characteristics of automation objects determine the initial and final dynamic modes of objects. The static characteristic of the wastewater neutralization process is the dependence of the $\mathrm{pH}$ value on the dose of the reagent in the steady state and in the automation process which is important. On the basis of static characteristics, the management process paradigm is determined and the structural-parametric scheme of the control system, including the feedback and the feedback structure, is substantiated. Also, in our technological calculations with the help of these characteristics we can determine correctly the reagent dose that is required for the wastewater neutralization process.

The second section of the article is devoted to the development of a mathematical model of statics of the buffer wastewater neutralization process; the third section describes the algorithm for calculating the total dose of the reagent for neutralizing buffer wastewater; in the fourth section - the results of checking the mathematical model for adequacy are given; the fifth section describes the possibility of using microprocessor technology for automatic control systems for the neutralization process.

\section{Mathematical model of statics of the wastewater neutralization process with the buffer properties}

To obtain an adequate mathematical model a good understanding of the process mechanism is requied, later the results of mathematical modeling can be an invaluable tool to the entire $\mathrm{pH}$ algorithm regulating 
procedure.

The buffer solution can be considered as a reservoir of ions, which, if necessary, are released in the reaction. In wastewater the buffering effect is observed in the presence of weak acids and bases, and also their salts. In this connection, for a more accurate determination, the researchers recommend an additional independent measurement, for example ion-selective electrodes [12]. The model being developed is intended for simulating the the wastewater neutralization process without such an experiment under the following assumptions.

1. When a mixture of strong and weak acids is titrated, the reagent is first used to neutralize the strong acid to the $\mathrm{pH}$ value, which determines the start of titration of the weak acid. Further, until the end of titration of a strong acid, the $\mathrm{pH}$ value remains practically unchanged, and then the titration of a weak acid begins (Fig.1).

2. The change in the volume of waste water during the titration practically does not take place.

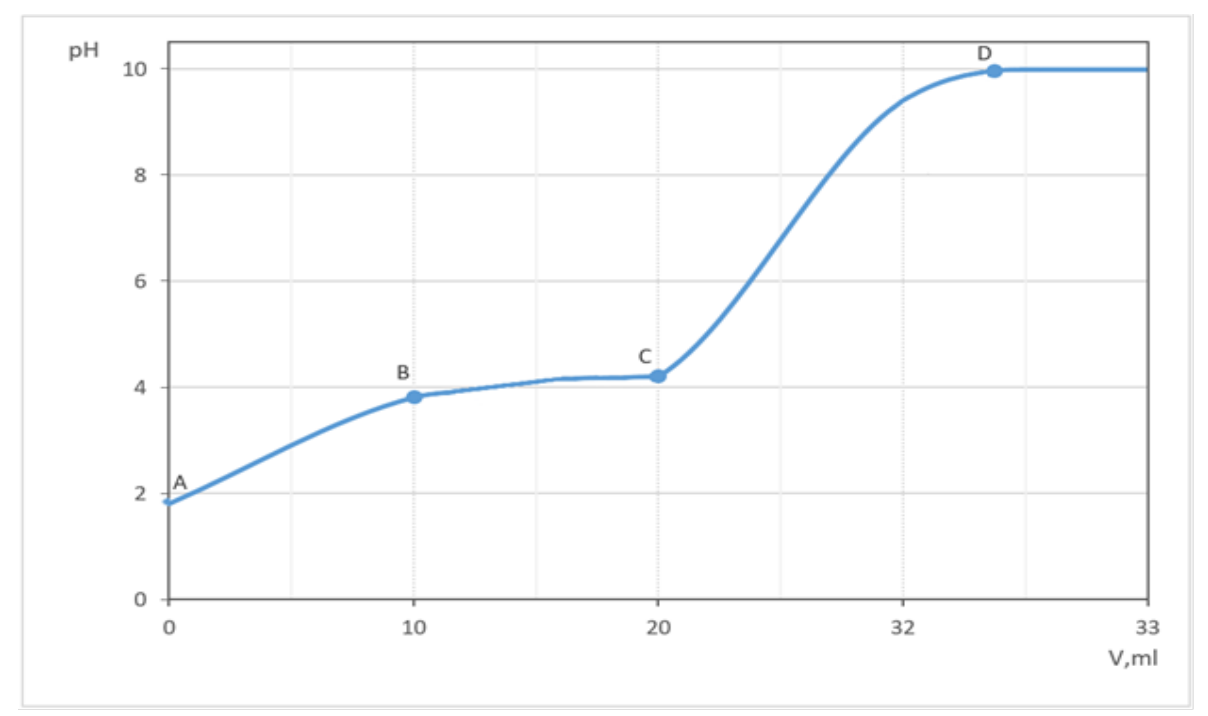

Fig.1. The stage of the process of wastewater neutralization having a strong and weak acid in their composition

Using the law of equivalents, and taking into account the second assumption, we define the amount of the reagent VTC, needed to achieve point $C$.

$$
V_{T C} C_{H T}=C_{K 1}+V_{K 1}
$$

whence it follows that $V_{T C}=C_{K 1} / C_{H T}$

where $C_{K 1}$ and $C_{H T}$ - are the molar concentration equivalents of a strong acid and reagent.

At point $\mathrm{A}$ the $\mathrm{pH}$ value is determined in the classical way:

$$
p H_{A}=-\lg \left(C_{K 1}\right)
$$

The amount of reagent required to reach point $\mathrm{B}$, may be founded in the following dependencies: 
$V_{T B}=\left(C_{K 1}-C_{K 1 S}\right) / C_{H T}$

where $C_{K 1 S}-$ is the molar concentration of a strong acid equivalent in the point B; $p H_{B}=p H_{C 1}$ (under the first assumption). To reach the point $\mathrm{D}$ equivalence the required amount of the reagent is defined as the sum.

$$
V_{T C}+V_{T 2}=V_{D}
$$

where $V_{T 2}=C_{K 2} / C_{H T}$,

$C_{K 2}$ - the molar concentration of the equivalent of a weak acid.

The functional dependence of the $\mathrm{pH}$ for points $\mathrm{C}$ and $\mathrm{D}$ has the following form:

$$
\begin{aligned}
& p H_{C}=-(1 / 2) p K_{a}-(1 / 2) \lg C_{a} ; \\
& p H_{D}=7-(1 / 2) p K_{a}+(1 / 2) \lg C_{c},
\end{aligned}
$$

where $K_{a}$ - the dissociation constant of a weak acid;

$C_{a}-$ the total molar concentration of a weak acid;

$C_{c}$ - the salt concentration in the solution.

Thus, the mathematical model of statics of the wastewater neutralization process can be written as a system of equations:

$$
\begin{array}{ll}
V_{B}=\left(C_{K 1}-C_{K 1 S}\right) / C_{T} ; \\
V_{C}=C_{K 1} / C_{T} ; \\
V_{D}=V_{B}+V_{C}+C_{K 2} / C_{T} ; \\
p H= \begin{cases}-\lg C_{K 1} & \text { when } V_{T}=0 \\
-(1 / 2) \lg K_{a}-2^{-1} \lg C_{a} & \text { when } V_{B} \leq V_{T} \leq V_{C} \\
-\lg C_{a}+\lg C_{c}-\lg K_{a} & \text { when } V_{C}<V_{T}<V_{D} \\
7-(1 / 2) \lg K_{a}+(1 / 2) \lg C_{c} & \text { when } V_{T}=V_{D}\end{cases}
\end{array}
$$

3. The automated calculation algorithm of the required reagent dose to neutralize the buffer wastewater

Mathematical modeling is nowadays characterised with the machine-oriented procedures formalization associated with the implementation of computer technology models. As already indicated, a large number of studies have been devoted to the problem of determination of $\mathrm{pH}$. The developed algorithm is one of several ways to solve the problem of determining the total dose of a reagent for neutralizing stubble waters containing strong and weak acids. Among other approaches, it has the advantage that it can be implemented on the computer equipment, since, as indicated earlier, no additional field experiment is required for this. The algorithm for static simulation of buffer wastewater neutralization is shown in Figures 2-5. 


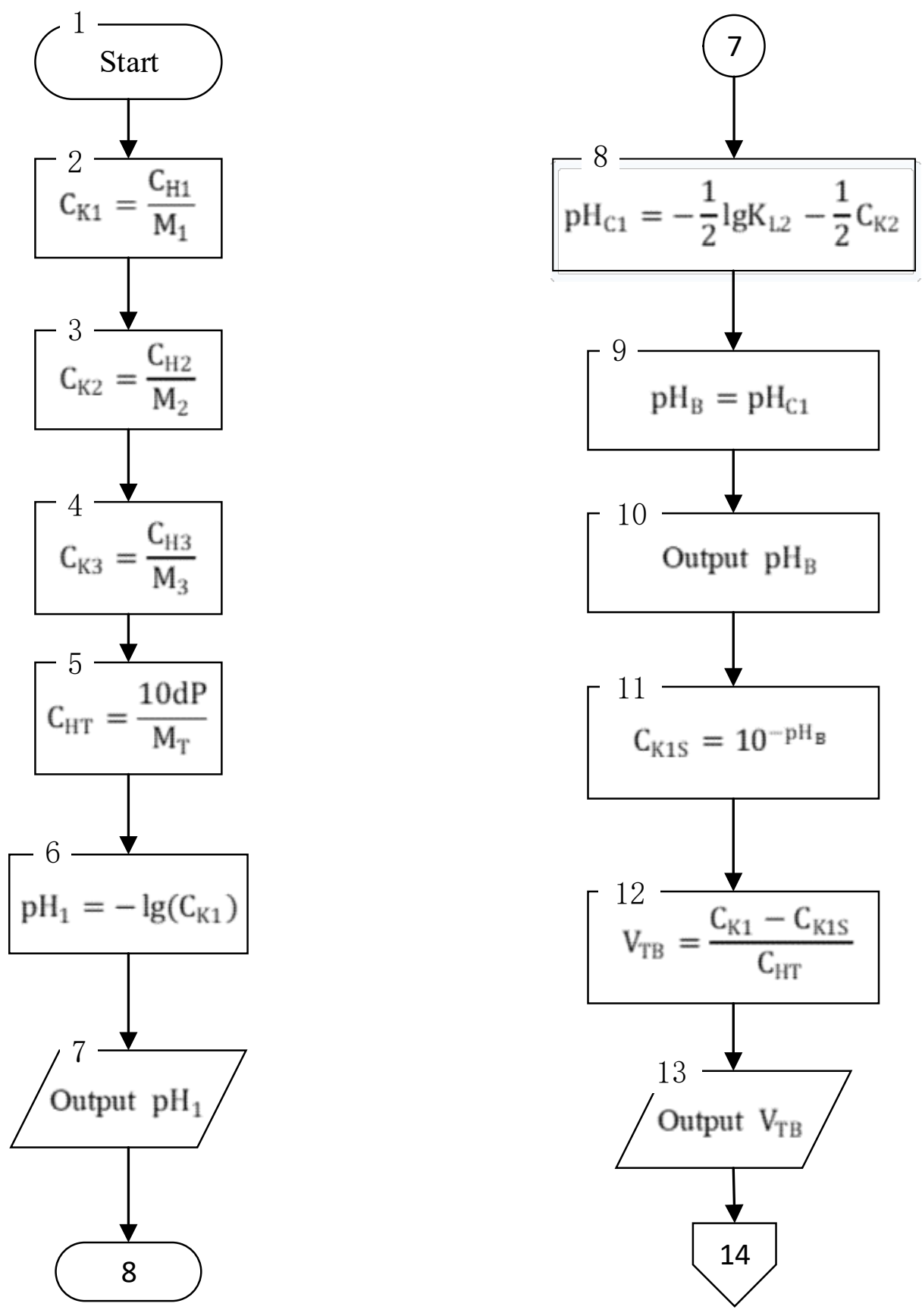

Fig.2. Determination of the amount of reagent to reach point B 

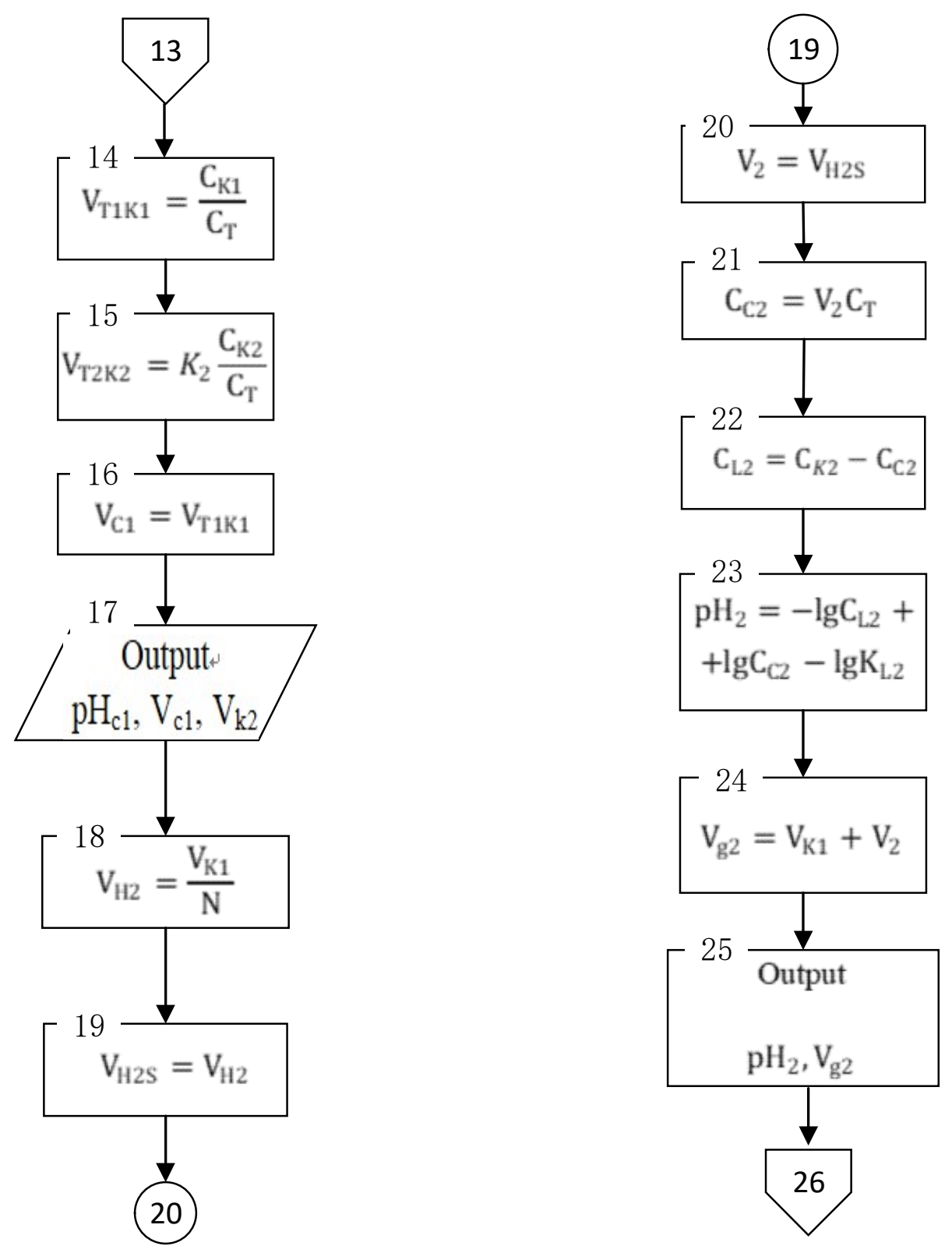

Fig.3. Determination of the amount of reagent to reach point $\mathrm{C}$ 

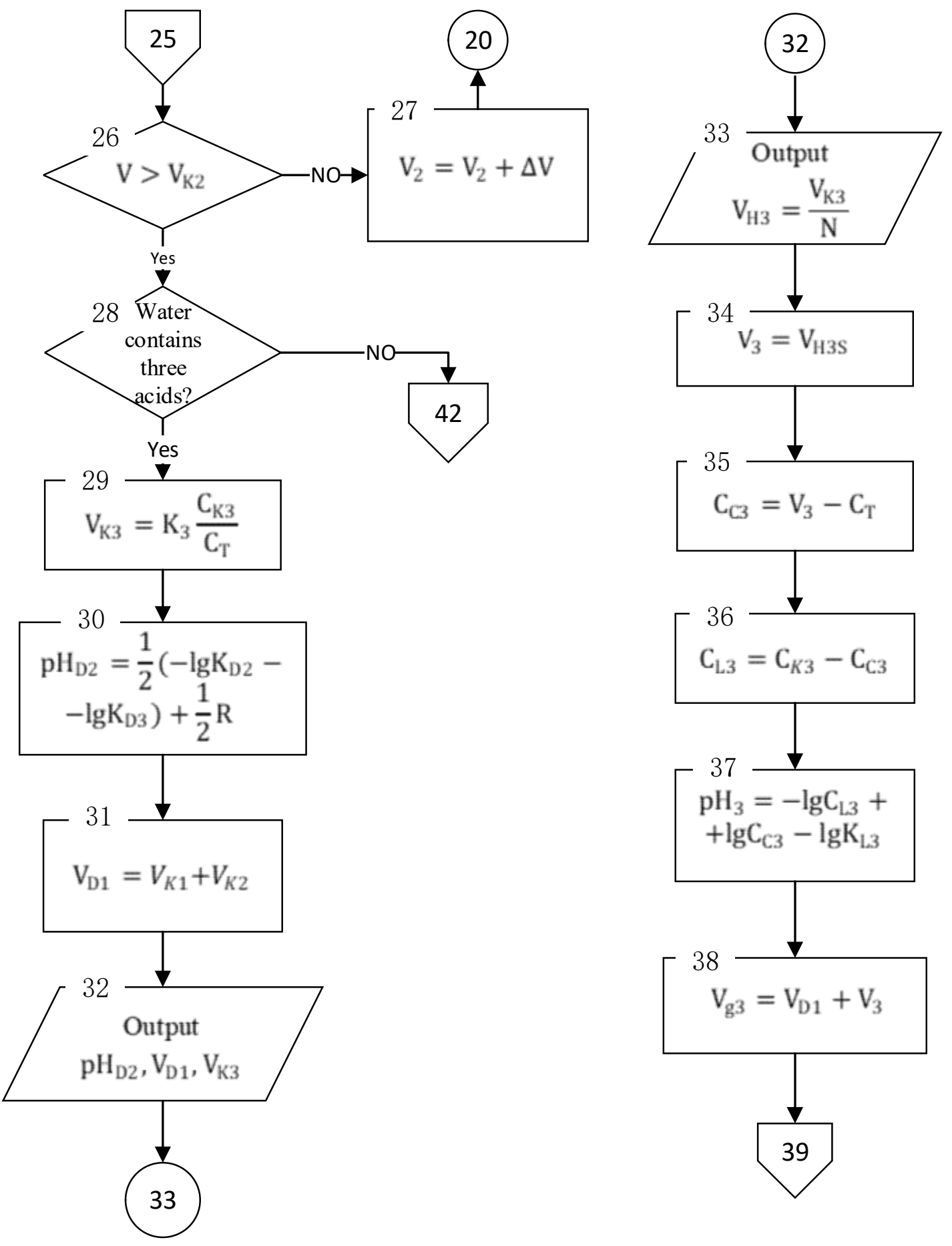

Fig.4. Determination of the amount of reagent to reach point D 


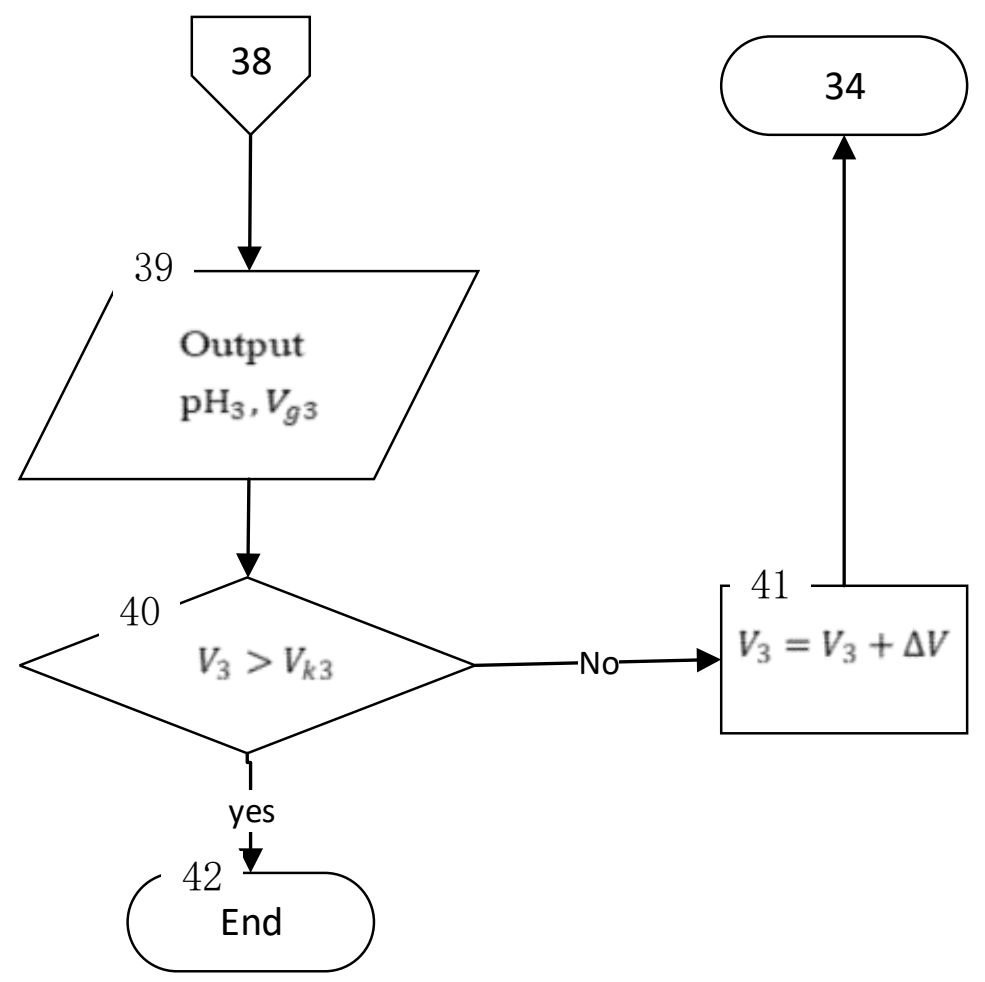

Fig.5. The output of total dose of the reagent

Block 1. The beginning.

Block 2. Calculate the transformed values of the strong acid initial concentration.

Block 3. Calculate the initial concentration transformed values of the first weak acid.

Block 4. Calculate the initial concentration transformed values of the second weak acid.

Block 5. Calculate the reagent concentration.

Block 6. Calculate the $\mathrm{pH}_{1}$ value in the first titration point.

Block 7. Derive the $p H_{l}$ value.

Block 8. Calculate the $\mathrm{pH}_{C l}$ value for point B.

Block 9. Assign to a variable $p H_{B}$ value $p H_{C I}$

Block 10. Derive the $p H_{B}$ value.

Block 11. Calculate the strong acid c oncentration for the point B.

Block 12. Determine the $V_{T B}$ reactant amount, needed to reach the point B.

Block 13. Derive the $V_{T B}$ value.

Block 14. Define the equivalent amount of reagent which is necessary to neutralize a strong acid.

Block 15. Define the equivalent amount of reagent which is required for the first weak acid neutralization.

Block 16. Assign to a variable $V_{C I}$ value $V_{K I}$.

Block 17. Derivate the values $\mathrm{pH}_{C 1}, V_{C 1}, V_{K 1}$

Block 18. Calculate the step for $V_{2} ; V_{H 2}=V_{K 1} / \mathrm{N}$.

Block 19. Assign to a variable $V_{\mathrm{H} 2 \mathrm{~S}}$ value $V_{H 2}$.

Block 20. Assign to a variable $V_{2}$ value $V_{\mathrm{H} 2 \mathrm{~S}}$.

Block 21. Determine the concentration of salt $C_{C 2}$. 
Block 22. Determine the concentration of the first weak acid undissociated portion.

Block 23. Calculate the titration $p H$ values for the first weak acid.

Block 24. Determine the $\mathrm{Vg}_{2}$ reactant amount for the first weak acid titration.

Block 25. Derive values $\mathrm{pH}_{2}$ and $\mathrm{Vg}_{2}$

Block 26. $V>V g_{2}$ ?

Block 27. $V_{2}=V_{2}+\Delta V$

Block 28. Analize the number of wastewater pollutants.

Block 29. Calculate the equivalent amount of reagent required for titrating the second weak acid.

Block 30. Calculate the $p H_{D I}$ value at the first equivalence point.

Block 32. Derive the values $p H_{D 1}, V_{D 1}, V_{K 3}$

Block 33. Calculate the step for $V_{3}, V_{H 3}=V k_{3} / \mathrm{N}$

Block 34. Assign to a variable $V_{3}$ initial value $V_{H 3 S}$

Block 35. Calculate the concentration of salt

Block 36. Calculate the concentration of the second weak acid undissociated portion.

Block 37. Calculate the titration $p H$ values for the second weak acid.

Block 38. Determine the reactant amount for the second weak acid titration.

Block 39. Derive values $\mathrm{pH}_{3}$ and $\mathrm{Vk}_{3}$

Block 40. $V_{3}>V_{K 3}$ ?

Block 41. $V_{3}=V_{3}+\Delta V$

Block 42. The End.

Based on the simulation results, the reagent total dose that is required for wastewater buffer neutralization is determined. The results presented in Fig. 6, are the good evidence that the experimental data consist with the $\mathrm{pH}$ values calculated from the model.

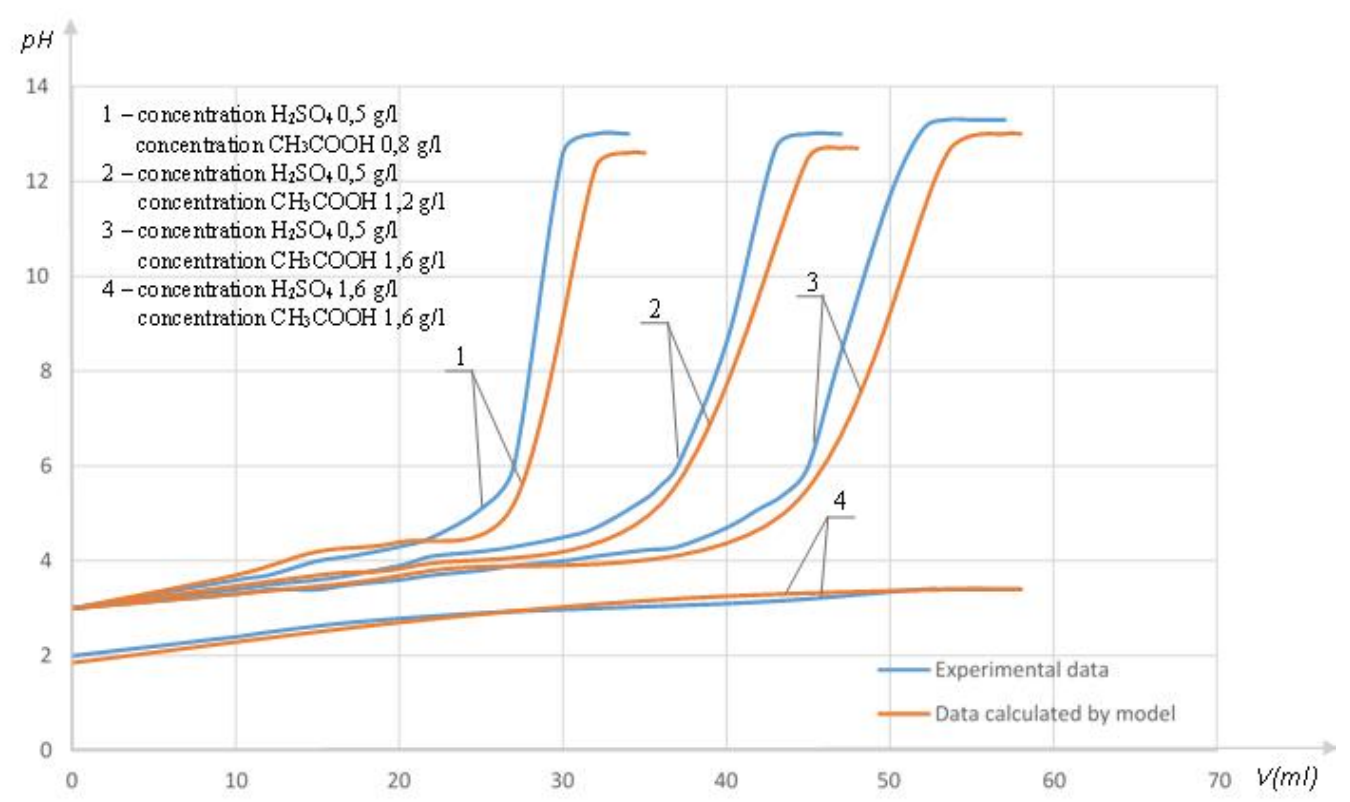

Fig.6. Comparison of experimental data with model data

The developed algorithm is implemented on the basis of microprocessor technology in the frames of $\mathrm{pH}$ 
adaptive cascade control (Fig.7).

\section{Checking the mathematical model for adequacy}

For this study, it is advisable to use statistical criteria as a criterion for the adequacy of the results of mathematical modeling for experimental data. The application of these criteria allows us to establish quantitative limits of adequacy. In this way we can more objectively determine the degree of identity of the object and its model.

The first method involves testing the hypothesis of the proximity of the mathematical expectations of each number of components of the model and the real system. It is as follows.

Conduct Ns experiments on a real object and receive for each number of components of the sample of values of the system. Then perform $\mathrm{Nm}$ experiments on the model and receive the same number of components of the sample of sample values of the model.

Usually trying to make the volumes of the samples identical $(\mathrm{Ns}=\mathrm{Nm})$, but in-person experiments are very expensive, so, usually, $\mathrm{Nm}>\mathrm{Ns}$.

After these stages, the Student's criterion to calculate and compare it with the tabular value. First needed the level of significance to set. Then according to the table of the Student's criterion determine its critical value $t_{k r}$. In the case when the condition $t_{k}<t_{k r}$ is fulfilled, the hypothesis about the proximity of mathematical expectations for the number of components of the model and the system is adopted and the model is considered adequate.

The second method involves checking the hypothesis of the homogeneity of the two dispersions $S_{k}^{2, s}$ and $S_{k}^{2, m}$ for each number of components. The parameter i $S_{k}^{2, m}$ is the variance of the model feedback with respect to the mean value of the system response.

This hypothesis is accepted in the case when the condition $F<F_{k r}$ is fulfilled, where $F$ and $F_{k r}$ are the calculated and critical values of Fisher's criterion.

After determining the selective value of Fisher's criterion, the table defines the critical value of criterion Fkr, using information on degrees of freedom kmore, kless and the level of significance $\alpha$.

Estimates of statistical characteristics for the data obtained during the experiment are given in Table 1. The level of significance is $\alpha=0,05$. Table 1 shows the statistical characteristics of the data.

Table 1. Statistical characteristics of data

\begin{tabular}{|c|c|c|c|c|c|}
\hline \multicolumn{2}{|c|}{ The number of experiments } & \multirow{2}{*}{$\begin{array}{c}\mathbf{N 1} \\
3\end{array}$} & \multirow{2}{*}{$\begin{array}{c}\mathbf{N 2} \\
4\end{array}$} & \multirow{2}{*}{$\begin{array}{c}\mathbf{N 3} \\
5\end{array}$} & \multirow{2}{*}{$\begin{array}{c}\mathbf{N 4} \\
6\end{array}$} \\
\hline 1 & 2 & & & & \\
\hline \multirow{2}{*}{$\begin{array}{c}\text { Statistical } \\
\text { characteristics of } \\
\text { the system }\end{array}$} & $\begin{array}{c}\text { Mathematical } \\
\text { expectation }\end{array}$ & 6,40833 & 6,205 & 6,09682 & 2,90909 \\
\hline & Dispersion & 15,74265 & 13,34786 & 15,01984 & 0,21491 \\
\hline \multirow{2}{*}{$\begin{array}{c}\text { Statistical } \\
\text { characteristics of } \\
\text { the model }\end{array}$} & $\begin{array}{c}\text { Mathematical } \\
\text { expectation }\end{array}$ & 6,075 & 5,8025 & 5,815909091 & 2,77 \\
\hline & Dispersion & 13,44023 & 11,33785 & 10,78452 & 0,3095 \\
\hline \multirow[t]{2}{*}{ Student's criteria } & Calculated & 0,05578 & 0,10278 & 0,07126 & 0,91011 \\
\hline & Tabular & 2,074 & 2,024 & 2,018 & 2,145 \\
\hline \multirow[t]{2}{*}{ Fisher's criteria } & Calculated & 1,17131 & 1,17728 & 1,39272 & 1,44014 \\
\hline & Tabular & 2,84 & 2,84 & 2,16 & 3,84 \\
\hline
\end{tabular}

As can be seen from Table 1, the calculated values of the Student and Fisher tests are less than tabular ones 
for all experiments. Thus, we can conclude that there is no reason to reject the hypothesis about the adequacy of the developed mathematical model. Therefore, we believe that the mathematical model adequately describes the statics of the technological process of neutralization of buffer sewage.

\section{The use of microprocessor equipment in systems of automatic control of the wastewater neutralization process}

Consequently, the process of wastewater neutralization, which has buffer properties, is related to automation objects with variable parameters and variable structure. Our small contribution to the simulation of this process is of great practical use in utilizing the microprocessor technology in $\mathrm{pH}$ control circuit, which the implementation of complex control algorithms is possible to be based on. The advantage of microprocessors is the ability to implement adaptive control algorithms that may not be implemented on regulatory, functional and analog blocks.

The analysis of the static characteristics of the neutralization process makes it possible to conclude that the principle of cascading adaptive control of this process is rational to implement. In this approach, after perturbation of the input parameters, adjusting of the coefficient of proportionality of the regulator in the control circuit will occur both by perturbation, and by a signal determined by the principle of PI in the feedback coupling loop. The specified correction of control influence is carried out with the help of the program, which is enrolled in auxiliary storage and is realized in the random-access memory of the microprocessor. The calculation of the total dose of the reagent for the neutralization of buffer wastewater is carried out in accordance with the algorithm given above. In Fig. 7 the principal scheme of such a control system at the technological level is shown ( $\mathrm{Q}$ - wastewater consumption).

Internal elements of the structure (microprocessor, random-access memory and auxiliary storage, interface of input and output) provide the connection of the microprocessor with the post of the operator-technologist, schemes of the signals digital values formation from sensors and control of the executive mechanism. Analog signals are routed through a switch and an amplifier to an analog and digital converter, where they are turned into digital suitable for processing by a microprocessor ones. The proposed structure has a universal mode. When a new type of sensor of a certain type is deployed, the software to provide its maintenance may be downloaded and used automatically if the system indicates that this sensor is of the appropriate class. In addition, by changing the process of software control the management algorithm may be complicated and improved without any hardware changes [14].

The operator-technologist has a monitor for managing the wastewater purification processes of the whole enterprise [15]. For typical wastewater purification processes on this monitor there are [16] :

- a line of input of the control mode operator;

- a line for entering intermediate variables related to the choice of this operator;

- a line of parameters of wastewater purification processes;

- a line of parameters for setting control modes;

- the fractional control effect for the wastewater purification process;

- the main rules governing the switching of control modes stored and downloaded;

- the adaptation of the top-level control modes;

- the connection to the database of the automated process control subsystem of water conservation.

\section{Summary}

It is very pleasant to realize that there are still complex processes that require a proper understanding and ability from a researcher who is trying to measure, model, and manage them. For researchers, the $\mathrm{pH}$-process 
and its regulation is an interesting and complex area. There is a wide variety of approaches for solving this problem and so far nobody can give a final solution and formulate the absolute truth, since each of its applications is unique. As indicated by the researchers Ylen J-P and Kazemian, H. B., in some individual cases, PID-algorithms [17,18] work properly. In our work cascade adaptive regulation of $\mathrm{pH}$ based on microprocessor technology is proposed, when the gain in the circuit of the actuator is adjusted both by intrusion of the input parameters and by the feedback signal, which is determined by the PI-algorithm.

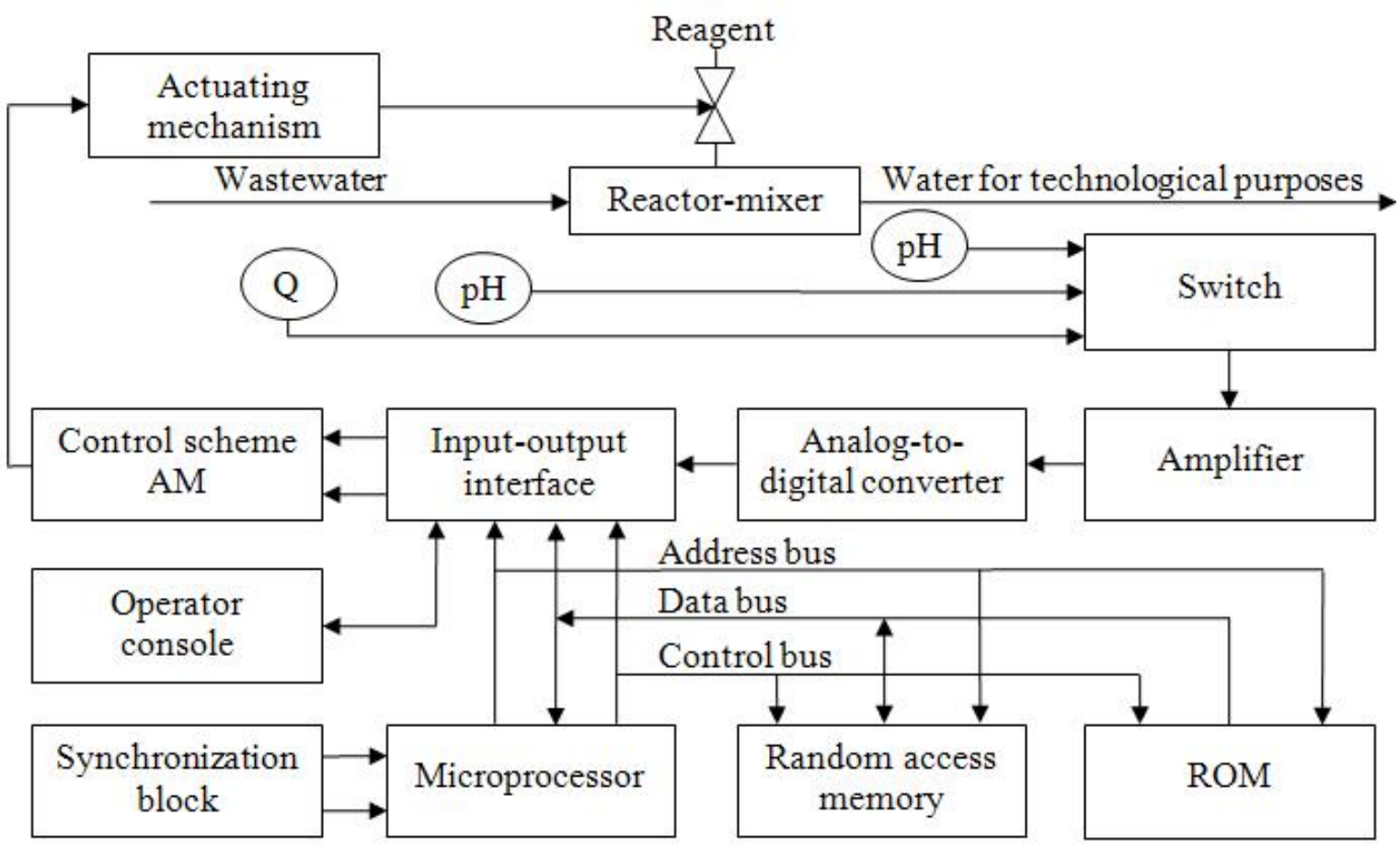

Fig.7. Schematic diagram of adaptive control system for the process of wastewater neutralization

Summarizing it is possible to say that the theoretical contribution of this work is the implementation of the principle of decomposition of the process of neutralizing buffer wastewater at the stage. These stages are characterized by different kinetic properties. The value of $\mathrm{pH}$ was determined for each stage separately. This approach allowed us to develop an appropriate algorithm for calculating the total dose of reagent for buffer wastewater neutralization.

The analysis of the static characteristics of the wastewater neutralization process has given the opportunity to develop the structural and parametrical scheme by the process of neutralization. The implemented algorithm for calculating the required doses of the reagent for buffering wastewater neutralization has been tested for microprocessor technology in the design of an automatic control system for wastewater purification at the Kiev Chemical Plant.

As it has been mentioned in the 1st chapter the wastewater treatment is mostly the complex of methods: filtration, neutralization, purification by storage and precipitation, bio-treatment, ion exchange etc. The automated control systems considered in this scholar paper can be used in the wastewater treatment plants' distributed control system (DCS). To achieve this goal joint DCS's network structure has to be developed. The analysis of available microcomputers' communication system with standard sewage treatment techniques 
and procedures has to be conducted. Then the new ones are to be developed if required. Subsequently the algorithms and software to control wastewater treatment procedure (which has not been in the focus of the research conducted) has to be designed.

\section{References}

[1] R. Connor, The United Nations world water development report 2015: water for a sustainable world. -UNESCO Publishing, 2015. -T. 1. -125 pages.

[2] S. Ortega Alba and M. Manana, Energy Research in Airports: A Review, MDPI, Energies, 9, $349,2016$. DOI:10.3390/en9050349

[3] I.C. Carvalho, M.L. Calijuri, P.P. Assemany, M.D.F.M.e. Silva, R.F. Moreira Neto, A.F. Santiago, M.H.B. de Souza, Sustainable airport environments: A review of water conservation practices in airports. RCRecycling 74, pp. $27-36,2013$. Available from Internet: http://dx.doi.org/10.1016/j.resconrec.2013.02.016

[4] A. Zhuchenko, M. Kvasko, R. Osipa "The use of microprocessor technology in wastewater treatment control systems". Bulletin of the National Technical University of Ukraine "Igor Sikorsky Kyiv Polytechnic Institute", ser." Chemical Engineering, Ecology and Resource Saving", Vol.2, No.8, pp. 143-145, 2011.

[5] Anatolii I. Zhuchenko, Liudmyla V. Osipa, Evgeniy S. Cheropkin,"Design Database for an Automated Control System of Typical Wastewater Treatment Processes", International Journal of Engineering and Manufacturing (IJEM), Vol.7, No.4, pp. 36-50, 2017. DOI: 10.5815/ijem.2017.04.04

[6] A. Bieliatynskyi, L. Osipa, L. Yaroshchuk, E. Cheropkin. Information support of automated control subsystem of the airport's water-saving processes // Proceedings of the 21th Conference for Junior Researchers "Science - Future of Lithuania": TRANSPORT ENGINEERING AND MANAGEMENT (Vilnius, 4-5 May 2018, Lithuania). - pp. 146-150. — Access mode: http://jmk.transportas.vgtu.lt/index.php/tran2017/tran2018/paper/viewFile/148/203.

[7] Stanley K, Gandhi S. "Industrial effluent water pH Neutralization controller design using LabVIEW \&Matlab." International Journal of Pure and Applied Mathematics. Volume 117, No. 10, pp. 19-23, 2017, ISSN: 1311-8080, doi: 10.12732/ijpam. v117i10.4

[8] Boozarjomehry, R. B., Svcek, W. Y., Output feedback neurolinearization, ISA Transactions, 40, pp. 139-154, 2001.

[9] Biagiola S. I., Agamennoni O. E., Figueroa J. L. Robust control of wiener systems: application to a $\mathrm{pH}$ neutralization process. Brazilian Journal of Chemical Engineering. São Paulo Jan. Mar. ISSN 0104-6632, vol. 33, No.1, pp. 4358-4383, 2016

[10] Deapali Gupta, Balwinder Singh, Harpreet Singh, "Design and Development of Pesticide Residue Detection System using EC and $\mathrm{pH}$ Sensor", International Journal Engineering and Manufacturing (IJEM), 2016, 2, pp.10-17. DOI: 10.5815/ijem.2016.02.02

[11] De, D. S., Loh, A.-P., Krishnaswamy, P. R., A nonlinear adaptive controller of pH neutralization process, Proc. Am. Contr. Conf, 2250-2254, Chicago, 2000.

[12] Franchuk G. M, Majd S. M ., Bondaruk A. M. "Comprehensive assessment of the quality of water ecosystems polluted wastewater discharges from the airport." Kyiv, National Aviation University, High technology, Vol. 2 (22), pp. 246-249, 2014. (In Ukrainian)

[13] Higgins P. Ion selective electrode measurement - fundamentals in online analysis. Available from Internet: $\quad<\quad$ https://www.ysi.com/ysi-blog/water-blogged-blog/2013/09/ion-selective-electrodemeasurement-fundamentals-in-online-analysis $>$.

[14] A. Bieliatynskyi, L. Osipa, A. Klochan, E. Cheropkin. Control algorithm of the airport's water-saving processes // Proceedings of the 21th Conference for Junior Researchers "Science - Future of Lithuania": TRANSPORT ENGINEERING AND MANAGEMENT (Vilnius, 4-5 May 2018, Lithuania). - pp. 
137-141. - Access mode: http://jmk.transportas.vgtu.lt/index.php/tran2017/tran2018/paper/viewFile/ $149 / 163$.

[15] Andrii Bieliatynskyi, Liudmyla Osipa and Bogdan Kornienko. Water-saving processes control of an airport, MATEC Web of Conferences , Vol. 239, 05003 ( 2018) DOI: https://doi.org/10.1051/matecconf/201823905003. - Access mode: https://www.matec-conferences.org/articles/matecconf/abs/2018/98/ matecconf ts2018 05003/matecconf ts2018 05003.html

[16] A. Zhuchenko, R. Osipa and L. Osipa, "Optimization tasks, which are solved in the integrated automated control system of water saving processes.", Bulletin of the National Technical University of Ukraine "KPI named after I. Sikorsky", ser." Chemical Engineering, Ecology and Resource Saving", - 2016. vol. 15 , no 1 , pp. 108-110.

[17] Ylén Jean-Peter. Measuring, modelling and controlling the $\mathrm{pH}$ value and the dynamic chemical state. Helsinki University of Technology Control Engineering Laboratory. 2001. - 145p. - Access mode: http://citeseerx.ist.psu.edu/viewdoc/download?doi=10.1.1.136.8092\&rep=rep1\&type=pdf

[18] Kazemian, H. B., Comparative study of a learning fuzzy PID controller and a self-tuning controller, ISA Transactions, 40, pp. 245-253, 2001.

\section{Author's Profile}

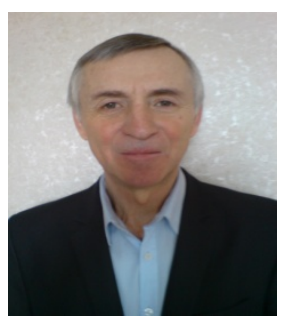

Ruslan A. Osipa (Ph. D.) is working as Assistant Professor of the Automation of chemical production department at National Technical University of Ukraine "Igor Sikorsky Kyiv Polytechnic Institute". His research interest includes automatic control system, control of complex chemical-technological systems, database, mathematical modeling and optimal process control. Author 51 research papers in reputed journals and conferences.

How to cite this paper: Ruslan A. Osipa," Statics Simulation of the Buffer Wastewater Neutralization Process ", International Journal of Engineering and Manufacturing(IJEM), Vol.9, No.4, pp.1-14, 2019. DOI: 10.5815/ijem.2019.04.01 